\section{Achieving Patient Compliance}

M. Robin DiMatteo and D. Dante DiNicola

Pergamon Press Inc., Elmsford, New York, 1982

Numerous publications and research articles on patient compliance have swelled the literature to the point of confusion. Many studies test one aspect of theory or one educational intervention without assessing its fit into the total picture or any theoretical framework. Achieving Patient Compliance is a somewhat successful attempt to synthesize compliance theory, research, and practice into one comprehensive model. The model is developed and described by a social psychologist (DiMatteo) and a physician (DiNicola) who began collaborative work after a "serendipitous meeting" because "the cages housing DiNicola's experimental pigs were leaking into DiMatteo's office, precariously close to the empirical data on patient-practitioner relationships...." Their collaborative effort resulted in a well-written and cohesive book as well as their marriage halfway through Chapter 4.

The book as a whole presents an excellent framework for understanding patients' and providers' behaviors and offers guidance for productive provider-initiated change as well as for further research in patient compliance. The basic philosophy and perspective of the authors respect the patient as being in control and responsible for health actions. The interaction and communication between provider and patient are appropriately regarded as the "most important link between patient compliance and the other factors that can affect it." The literature on compliance is thoroughly reviewed and discussed in terms of the authors' explanatory model.

After a chapter introducing the issues in compliance and the authors' philosophy, two chapters deal with practitioner-patient relationships, discussing the communication of information, rapport, and the art of medicine. Chapter 4 covers patients' perceptions of illness, prevention, and treatment using Ajzen and Fishbein's theory of reasoned action. Predictors such as locus of control and health belief model are discussed in the context of the Ajzen and Fishbein model. Chapter 5 presents the effect on norms and compliance of the patient's culture, especially social class, ethnic group, and family. Chapter 6 brings together an analysis of patient beliefs and social norms. The literature on changing beliefs, attitudes, and subjective norms is reviewed and the formation of behavioral intentions is described. Chapter 7 discusses methods that may be useful to enhance supports and overcome barriers in carrying out health behaviors. The 8th and final chapter of the book discusses ethical issues, roles, and negotiation between patient and practitioner, and the contractual model of joint cooperation between patient and practitioner in medical care. Although the book is written to be read as a whole and not used primarily as a reference text, lengthy bibliographies for each chapter as well as an author index enhance its utility.

Achieving Patient Compliance is an important contribution to the literature on patient compliance. The authors' comprehensive model of compliance serves as a useful way to organize and present an overview of the related theoretical and research literature. While every study is not always well described, the authors do interpret where conclusions can be drawn and where further research is needed. Practitioners will benefit from the broad approach to the understanding of compliance as well as the descriptions of specific strategies to improve compliance. The focus on the practitioner-patient relationship and interpersonal influence is also important. Although the authors state that their model is broadly applicable to preventive health behaviors as well as sick-role behaviors, the focus of the book is really on the doctor-patient relationship in situations in which the patient has a diagnosed medical condition. It is in this area of sick-role behavior that most of the research has been done, and the practical clinical examples of the authors follow suit. Although the practitioner who uses this book can be any of a number of health professionals, the physician seems to be the practitioner most often alluded to. Attention to the roles and practice of various other disciplines might have been helpful. Unfortunately, the tasks of health education as a discipline, in fact, are narrowly defined as primarily the provision of information to patients.

Overall, Achieving Patient Compliance is a pleasure to read. It is well organized, even, and coherent, in contrast to several edited books on patient compliance that are not only uneven but often lack a cohesive framework. The book is well balanced between the compliance problem and possible solutions. While of interest to researchers, the book's greatest use will be to students and practitioners, for it provides a well documented and referenced background for understanding patient behavior. Further, it offers a refreshing and optimistic view of how health-care providers can improve their interactions with patients and afford patients opportunities for self-directed change.

Jean Thatcher Shope, Ph.D.*
Department of Health Behavior
and Health Education
School of Public Health
University of Michigan

\footnotetext{
* Member of the Special Patient Education Book Review Committee composed of members of the Michigan Society for Health Care Manpower Education and Training and the Great Lakes Chapter, Society for Public Health Education.
} 